\title{
The Association between Depression and Suicidal Ideation among Postgraduate University Students
}

\author{
Dr. Satyananda Panda ${ }^{1}$ *
}

\section{ABSTRACT}

Suicide is one of the leading causes of death in university students and is often associated with depression. This study examined the associations between depression and suicidal ideation among male and female postgraduate university students. This study utilized a survey using simple random sampling methodology involving 60 respondents (30 male and 30 female) chosen at random from different streams of Sikkim University, India. The participants were assessed by using Beck Suicidal Ideation (BSI) and Beck Depression Inventory (BDI-II).The data were analysed by using mean, standard deviation and descriptive statistics -Pearson method of correlation and non-parametric statistics like t-test. The findings indicated that suicidal ideation was negatively associated with depression, and no significant gender difference was found on depression. Reviewed research revealed similar suicidal risk factors for males and females. However, the prevalence and strength of prediction of certain risk factors were found to vary gender-specifically. These findings support the utility of gender-sensitive suicide assessment, prevention and intervention strategies. It was recommended that suicide prevention programs, counselling centers, and psychology clinics on university campus should include suicide attitudes such as depression, anxiety, and stress in their assessment and identification of individuals who may be at risk for engaging in suicide behaviour.

Keywords: Depression, Suicidal Ideation, Gender, University Students, Therapy.

Suicide is the third leading cause of death among young people; however the incidence of attempted suicide is much more. It has been observed that after puberty, rate of suicide increases with age until it stabilizes in young adulthood. This increase in suicide may be associated with the onset and increase in depressive and other disorders during adolescents as compare to childhood (Gould et al., 1996) as well as greater suicidal intent with age (Brent et al., 1999). As compare to adult males, adolescent males complete suicide at rates approximately five times higher than rates for adolescent's females (Kochanek et al., 2004).

Kessler, Borges \& Walters (1999) reported that rates of attempted suicide rise precipitously during adolescence. Borst, Noam \& Bartok (1991) postulate that with the advent of puberty, social-cognitive changes lead to more internal than external attributions of unhappiness. This

\footnotetext{
${ }^{1}$ Assistant Professor, Department of Psychology, Sikkim University, Gangtok (Sikkim) - India *Corresponding Author

(C) 2015 I S Panda; licensee IJIP. This is an Open Access Research distributed under the terms of the Creative Commons Attribution License (http://creativecommons.org/licenses/by/2.0), which permits unrestricted use, distribution, and reproduction in any Medium, provided the original work is properly cited.
} 
shift in attributional style leads to more self-blame in response to interpersonal stressors and in some adolescents results in suicidal behaviour. Similarly, maladaptive cognitive processes frequently play an important role in suicidal behaviour. Although numerous studies have demonstrated a relation between hopelessness and adolescent suicide attempts, hopelessness does not consistently predict suicidality once depression is controlled (Esposito et al., 2003). It has been suggested that hopelessness may place adolescents at risk for suicidal behaviour for only a limited period during a depressive episode (Dori\&Overholser, 1999).

The relationship of suicide, depression, and other mental illnesses to the abuse of alcohol and other drugs should be given serious attention. An analysis of data from the NCHRBS found that students who reported suicidal ideation were significantly more likely than other students to carry a weapon, engage in a physical fight, boat or swim after drinking alcohol, ride with a driver who had been drinking alcohol, drive after drinking alcohol, and rarely or never use seat belts (Barrios et al., 2000).

\section{Depression and Suicidal Ideation}

Depression is frequently associated with suicidal ideation and suicidal behaviour in adolescents (Brand et al., 1994; Brent et al., 1999). The depression-suicidal ideation link has also been documented in research studies conducted with Asian adolescents. In a sample of more than 9,000 Korean high school students, Juon, Nam and Ensminger (1994) found depression to be the strongest and most consistent predictor of suicidal behaviors; students who had high scores on depression were 5.31 times more likely to report suicidal ideas and 3.19 times more likely to attempt suicide, as compared to those with low scores.

Large-scale epidemiological studies have found that before adolescence rates of clinical depression are similar among boys and girls (Nolen-Hoeksema, Girgus, \&Seligman, 1991). During adolescence, the prevalence of depression among females increases markedly, while the rise for males is more modest. The female: male ratio for clinical depression changes from approximate equivalence in childhood, to a female preponderance of 2:1 and this approximate ratio continues into adulthood (Allgood-Merten, Lewinsohn, \& Hops, 1990).

Studies using self-report measures have also found that females report higher mean levels of depressive symptomatology and a greater proportion of females will score above standard cut-off points for identifying depression compared with males (Garrison et al., 1989).

Alternatively gender itself might increase the risk for suicidal ideation quite independently from levels of depression.For example, even with the same level of depression females may be at greater risk of suicidal ideation than males. Indeed some commentators have speculated that females are more likely than males to express ideation in response to even quite low levels of depressive symptomatology and that this trend is more evident among young people in developed societies (Canetto,1997). 
Rates of suicidal ideation in adolescents have also not been nationally quantified in the United States. In one of the best studies, however, Lewinsohn and colleagues collected extensive data on suicidal behaviour in a large U. S. community sample $(n=1710)$ of primarily Caucasian adolescents (Andrews \&Lewinsohn, 1992; Lewinsohn, Rohde, \&Seeley, 1996). Contrary to Crosby and colleagues' findings, they found sex differences in rates of suicide ideation. The one point- in-time incidence of suicide ideation was significantly higher in girls (6.0\%) than in boys (2.3\%). In addition, the lifetime occurrence of suicidal ideation was significantly more common in young women (23.7\% of the girls) than in young men (14.8\% of the boys). With a few exceptions (e.g., Wright, 1985; Langhinrichsen-Rohling et al., 1998), this sex difference in adolescent ideation has typically been replicated in other samples of United States' adolescents and young adults (e.g., Lessard\&Moretti, 1998; Roberts, Chen, \& Roberts, 1997; Velez \&Cohen, 1988). These sex-specific findings have also begun to be replicated internationally. For example, Chinese adolescent females report more suicide ideation than Chinese adolescent males (Stewart et al., 1999).

\section{Rationale of the Study}

Suicide and suicide attempt is currently a major problem among students in our society, because of the inability to identify most of the risk factors in individuals with suicide ideation. In addition, in many developing countries, attempted suicide remains a punishable offence. Furthermore, in many places, injuries do not need to be reported and information on them is consequently not collected at any level. Other factors can also affect reporting, such as age, method of attempted suicide, culture and accessibility to health care. In short, the scale of attempted suicide is not clearly known for most countries. Therefore, it becomes increasingly difficult to intervene in suicide ideas and attempts, due to these difficulties in information collection. Death is no doubt one of the most painful realities of life. The death of someone close leaves both a physical and emotional void that provokes profound feelings of grief, loss, and anger among those who survive (Berman, Jobes\& Silverman, 2006). For most youth, however, death happens far away, or at some future time, or to others, especially those who lead lives of excessive risk. Thus, in a predominantly youth-oriented culture, particularly among the youth of that culture, death is a topic easily avoided or denied. It is in this context that the death of a young person hurts our sensibilities, especially when that death is self-imposed (Berman \& Carroll, 1984).It is in this context that the suicide of a young person hurts the lives of peers and loved ones.

It is against this backdrop that this study sets out to investigate the associations between depression and suicidal ideation among postgraduate university students.

\section{HYPOTHESES}

H1: There would be significant and positive correlation between depression and suicidal ideations among post-graduate university students.

H2: The depression level of female post-graduate students would be higher than the male postgraduate students. 
H3: There would be significant gender differences on the variable of suicidal ideation.

\section{METHODS}

\section{Participants}

The present study utilized a survey using simple random sampling methodology involving 60 respondents (30 male and 30 female) in the age group of 21-24 years chosen at random from different disciplines of Sikkim University, India. Student participation was voluntary, and none received compensation for taking part in the study. None of the participants suffered from any type of personality/psychiatric disorder or organic brain lesions.

\section{Tools Used}

The following psychological tools were used for the purpose of collection of data:

1. The Beck Depression Inventory (Beck, Steer \& Brown, 1996): The Beck Depression Inventory is a widely utilized 21-item self-report scale in both clinical and research studies. The Beck Depression Inventory-II is a depression rating scale that can be used in individuals that are ages 13 years and older, and rates symptoms of depression in terms of severity on a scale from 0 to 3 based on the 21 specific items. The test-retest reliability for BDI-II was 0.93 .

2. Beck Scale for Suicidal Ideation (Beck and Steer, 1991): Beck Scale for Suicidal Ideation (SSI) is a 21-item self -report instrument that clinicians can use to detect and measure the severity of suicidal ideation in adults and adolescents. The SSI is a clinical rating instrument which has been used since 1970 for assessing suicidal ideation in psychiatric patients. The BSS measures a broad spectrum of attitudes and behaviours that clinicians routinely consider when assessing patient suicide risk; it also reveals specific suicidal characteristics which require greater clinical scrutiny.

\section{Procedure}

Researcher explained the purpose of the study and explicitly sought the consent of the students regarding participation, as well as to ensure that students' responses were kept either anonymous or confidential. The purpose of the study was clearly explained to the students and consent to participate in the study was obtained from all students involved. Participation was strictly voluntary and students' responses were kept anonymous as they were instructed not to write their names. Students were also informed that they could refuse or discontinue participation at any time. These tests were administered individually following the instructions specified in the respective test manual. The general testing conditions were satisfactory and the procedure was uniform all through. All the tests were scored as per the procedure described in respective test manual.

\section{Data Analysis}

The descriptive analysis of the data was computerized and analysed using the Statistical Package for the Social Sciences (SPSS) version 22. The quantitative variants were given in descriptive statistics and the regularity checking was done by means of statistic testing while the qualitative variants were described by frequencies. The significance of the association between continuous variables was tested using Pearson's and Spearman’s correlation coefficient. 


\section{RESULTS AND DISCUSSION}

Table 1 represents the means, standard deviations and correlations of the key study. It is worth noting here that a non-significant and negative correlation has been found between suicidal ideation and depression.

Table 1: Mean, SD and Correlation of Depression and Suicidal Ideation of Postgraduate University Students $(N=60)$

\begin{tabular}{|l|l|l|l|l|l|l|}
\hline $\begin{array}{l}\text { Sl. } \\
\text { No. }\end{array}$ & Variables & N & Mean & SD & $\mathbf{1}$ & $\mathbf{2}$ \\
\hline 1 & Depression & 60 & 15.92 & 7.72 & 1.00 & -.021 \\
\hline 2 & Suicidal Ideation & 60 & 9.78 & 5.34 & -.021 & 1.00 \\
\hline
\end{tabular}

This clearly outlines that if post-graduate university students will have depression, it is not necessary that they will also have suicidal ideations. However, earlier studies show that depression is a long-established risk factor for suicide (Brent et al., 1988; Driessen et al., 1998; Friedmanet al., 1983; Kessler, Borges \& Walters, 1999; Yen et al., 2003), and mood disorders are most frequently associated with suicide and suicide ideation and discussed as risk factors for the presence of suicidality (Chioqueta \& Stiles, 2003; Hawton, 1987). Therefore, the first hypothesis which states that "there would be significant and positive correlation between depression and suicidal ideations among post-graduate university students” is not accepted.

Table 2: Mean, SD and t-test of Postgraduate Students on Depression $(N=60)$

\begin{tabular}{|l|l|l|l|l|l|}
\hline Variables & \multicolumn{1}{|c|}{$\mathbf{N}$} & \multicolumn{1}{|c|}{ Mean } & \multicolumn{1}{|c|}{ SD } & \multicolumn{1}{|c|}{ t-test } & Sig./ Non-sig. $(\mathbf{d f}=58)$ \\
\hline Male & 30 & 15.33 & 4.85 & \multirow{2}{*}{0.58} & Not significant \\
\hline Female & 30 & 16.5 & 9.83 & & \\
\hline
\end{tabular}

From Table 2 it can be observed that there is no significant difference between post graduate male and female students on depression. Therefore, the second hypothesis which states that "the depression level of female post-graduate students would be higher than the male post-graduate students" is not accepted. However, the mean score of female students is more than the mean score of male post graduate students on depression. This is in consistent with previous studies (Nerdrum et al., 2006; Herrero \& Meneses, 2006; Khawaja \& Duncanson, 2008).This result was inconsistent with the findings of other studies which reported that male students had higher levels of depressive symptoms than female students (Talaei et al., 2009), and other findings which found that no significant differences between males and females in depressive symptoms. 
The Association between Depression and Suicidal Ideation among Postgraduate University Students

Table 3: Mean, SD and t-test of Postgraduate Students on Suicidal Ideation $(N=60)$

\begin{tabular}{|l|l|l|l|l|l|}
\hline Variables & $\mathbf{N}$ & Mean & SD & t-test & Sig./ Non-sig. (df=58) \\
\hline Male & 30 & 0.3 & 0.46 & \multirow{2}{*}{0.52} & Not significant \\
\hline Female & 30 & 0.24 & 0.43 & & \\
\hline
\end{tabular}

It can be observed from Table 3 that there is no significant difference between post graduate male and female students on suicidal ideation. The third hypothesis which states that "there would be significant gender differences on the variable of suicidal ideation" is not accepted. However, the mean score of male students is more than the mean score of female post graduate students on suicidal ideation. Result of the present study is inconsistent with the earlier findings which have demonstrated that levels of depressive symptomatology and suicidal ideation are higher in females compared with males. It is also known that depression and suicidal ideation are strongly associated. The results of the present study are inconsistent with earlier findings.

\section{CONCLUSION}

Suicide is a serious issue which has to be dealt with immediately. According to estimates released by the World Health Organization (2010), suicidal attempts happen every three seconds, whereas deaths due to suicide happen every forty seconds which makes up to almost a million deaths connected to suicide every year. Committing suicide and depression are issues closely related with individual's mental health. Research done among college students show that suicidal ideation is not at a critical level. However, there is still tendency to think towards it based on the scores of respondents' answers. Moreover, it can be deduced that depression has a significant relationship with suicidal ideation. Therefore, in trying to carry out any form of intervention, depression should be given appropriate attention and should be prevented before it gets worse and eventually translated into suicidal actions. Counselling approach focusing on discussion and crises management is an alternative in inducing change, stability and in reducing risks of individuals who are having ideas of hurting themselves or committing suicide (Pelling, Bowers \& Armstrong, 2007). In dealing with suicide issues, all parties including parents, teachers, the government, media and non-governmental organizations (NGOs) must put in a concerted effort in order to solve this problem.

\section{INTERVENTIONS}

- Efforts to promote mental health and prevent suicide in colleges and universities must respond to the needs of each campus and its student population.

- The increasingly diverse atmosphere of higher education campuses presents challenges for preventing suicides and meeting the mental health needs of students.

- A comprehensive approach to suicide prevention on college and university campuses should employ multiple strategies targeted at both the general campus population and identifiable atrisk populations (Surgeon General of the United States, 1999). Such a comprehensive approach will be more effective when it includes consistent and coordinated activities in all 
the social spheres in which the target audience (in this case, college students) live, study, work, and play.

- A coordinated approach needs to engage key players in the college community in a planning process that focuses on assessment, design, implementation, and evaluation of suicide prevention activities. This effort provides a sound basis for considering a similar, customized approach for college and university communities.

- Elements of a comprehensive suicide prevention program include leadership to promote mental health and suicide prevention, screening, crisis management, educational programs, mental health services, life skills development, means restriction, social marketing, and social network promotion (NMHA \& Jed, 2002).

Some of the psychotherapeutic techniques can be provided are:

- Cognitive-behaviour therapy: In CBT, therapists focus on the impact a patient's present dysfunctional thoughts have on current behaviour and future functioning.

- Nondirective supportive therapy: Nondirective therapy defined as any unstructured therapy without specific psychological techniques other than those common to all approaches, such as helping people to ventilate their experiences and emotions and offering empathy.

- Psychodynamic therapy: The primary objective in (short-term) psychodynamic therapy is to enhance the patient's understanding, awareness, and insight about repetitive conflicts (intrapsychic and intrapersonal).

\section{LIMITATIONS AND FUTURE DIRECTIONS}

The scope of the present study is delimited to understand the suicidal ideation and depression among the university students. The students who participated in the screenings of this study were self-referred; therefore, students who are struggling with the greatest burden of depressive symptoms may not have participated. As such, our findings may actually underestimate the current degree of depressive symptoms and suicidality on university campuses.

Following suggestions in the form of future directions may be laid down in the continuity of the present study and in light of the delimitation of the study:

- The present study was conducted on university students, similar studies can be carried out to study relationship of depression and suicidal ideation at other level of education.

- The present study can be conducted on large sample for more reliable results.

- The study can be further extended to find out the difference between depression, suicidal ideation, socio-economic condition, academic anxiety, stress, family condition and considering many more other variables.

- Moreover, future research could use in-depth suicide assessment/ measures, especially to assess more complex relationships among different aspects of suicidality.

- Lastly, future interventions may benefit from targeting co-morbid symptoms of anxiety and depression to reduce suicide ideation and suicide attempts 


\section{REFERENCES}

Allgood-Merten, B., Lewinsohn, P.M., Hops, H. (1990). Sex differences and adolescent depression. Journal of Abnormal Psychology, 99, 55-63.

Andrews, J.A., \&Lewinsohn, P.M. (1992). Suicidal attempts among older adolescents: Prevalence and co-occurrence with psychiatric disorders. Journal of the American Academy of Child and Adolescent Psychiatry, 31, 655-662.

Barrios, L.C., Everett, S.A., Simon, T.R., \&Brener, N.D. (2000). Suicide ideation among US college students: Associations with other injury risk behaviors. Journal of American College Health, 48 (5), 229-233.

Beck, A.T. \& Steer, R.A. (1991). Manual for the Beck Scale for Suicide Ideation. San Antonio, TX: Psychological Corporation.

Beck, A.T., Steer, R.A., \& Brown, G. (1996). Manual for the Beck Depression Inventory-II. San Antonio, TX: Psychological Corporation.

Beck, A.T., Steer, R.A., \&Ranieri, W.F. (1988). Scale for suicide ideation: Psychometric properties of a self-report version. Journal of Clinical Psychology, 44, (4), 499-505.

Berman, V. \& Carroll, S. (1984). An analysis of suicidal ideation in a college sample. Archives of Suicide Research, 11, 41-56.

Berman, V., Jobes, A. \& Silverman, B. (2006). Attitudes toward suicidal behavior: A review of the literature. Death Studies, 16, 31-43.

Borst, S., Noam, G., \& Bartok, J. (1991). Adolescent suicidality: a clinical developmental approach. Journal of the American Academy of Child and Adolescent Psychiatry, 30, 796-803.

Brand, E.F., King, C.A., Olson, E., Ghaziuddin, N., \& Naylor, M. (1996). Depressed adolescents with a history of sexual abuse: Diagnostic co-morbidity and suicidality. J Am Acad Child AdolescPsychiatr35, 34-41 20.

Brent, D.A., Baugher, M., Bridge, J., Chen, T., \&Chiapptta, L. (1999). Age- and sex-related risk factors for adolescent suicide. J Am Acad Child AdolescPsychiatr, 38, 1497-1505.

Brent, D.A., Perper, J.A., Goldstein, C.E., Kolko, D.J., \& Allan, M.J., (1988). Risk factors in adolescent suicide: A comparison of adolescent suicide victims with suicidal in-patients. Archives of General Psychiatry, 45, 581-588.

Canetto, S.S. (1997). Meaning of gender and suicidal behavior during adolescence. Suicide and Life Threatening Behavior, 27, 339-351.

Chioqueta, A.P., \& Stiles, T.C. (2003). Suicide risk in outpatients with specific mood and anxiety disorders. Crisis, 24, 105-112.

Dori, G. \&Overholser, J. (1999). Depression, hopelessness, and self- esteem: accounting for suicidality in adolescent psychiatric patients. Suicide and Life-Threatening Behaviour, 29, 309-318.

Driessen, M., Veltrup, C., Weer, J., Joh, U., Wetterling, T., \&Dilling, H. (1998). Psychiatric comorbidity, suicidal behaviour and suicidal ideation in alcoholics seeking treatment. Addiction, 93, 889-894. 
Esposito, C., Johnson, B., Wolfsdorf, B.A., \&Spirito, A. (2003). Cognitive factors: Hopelessness, coping, and problem solving. In A. Spirito\& J. Overholser (Eds), Evaluating and Treating Adolescent Suicide Attempters: From Research to Practice (pp. 89-108). New York: Academic Press.

Friedman, R.C., Aronoff, M.S., Clarkin, J.F., Corn, R., \& Hurt, S.W. (1983). History of suicidal behavior in depressed borderline inpatients. American Journal of Psychiatry, 140(8), 1023-1026.

Garrison, C. Z., Schluchter, M. D., Schoenbach, V. J., \& Kaplan, B. K. (1989). Epidemiology of depressive symptoms in young adolescents. Journal of the American Academy of Child and Adolescent Psychiatry,28, 343-351.

Gould, M.S., Fisher, P., Parides, M., Flory, M., \& Shaffer, D. (1996). Psychosocial risk factors of child and adolescent completed suicide. Archives of General Psychiatry, 53, 155-162.

Hawton, K. (1987). Assessment of suicide risk. British Journal of Psychiatry, 150, 145-153.

Herrero, J.\&Meneses, J. (2006). Short Web-based versions of the perceived stress (PSS) and Center for Epidemiological Studies-Depression (CESD) Scales: A comparison to pencil and paper responses among Internet users. Computers in Human Behavior, 22, 830-846.

Juon, H., Nam, J. J., \&Ensminger, M. E. (1994). Epidemiology of suicidal behavior among Korean adolescents. J Child PsycholPsychiatr Allied Disciplin, 35, 663-677.

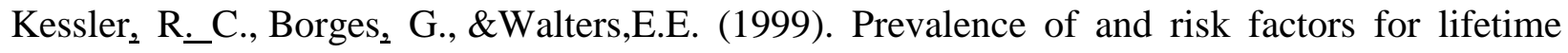
suicide attempts in the National Co-morbidity Survey.Arch Gen Psychiatry,56(7):617-26.

Kessler, R.C., Borges, G., \& Walters, E. E. (1999). Prevalence of and risk factors for lifetime suicide attempts in the National Co morbidity Survey. Archives of GeneralPsychiatry, 56, 617-633.

Khawaja, N. G. \&Duncanson, K. (2008) Using the University Student Depression Inventory to Investigate the Effect of Demographic Variables on Students' Depression. Australian Journal of Guidance and Counselling, 18(2), 1-15.

Kochanek, K.D., Murphy, S.L., Anderson, R.N., \& Scott, C. (2004). Deaths: Final data for 2002. National Vital Statistics Reports, 53(5). Hyattsville, MD: National Center for Health Statistics.

Langhinrichsen-Rohling, J., Lewinsohn, P., Rohde, P., Seeley, J., Monson, C. M., Meyer, K. A., \& Langford, R. (1998). Gender differences in the suicide-related behaviors of adolescents and young adults. Sex Roles, 39, 839-854.

Lessard, J.C. \&Moretti, M.M. (1998). Suicidal Ideation in an Adolescent Clinical Sample: Attachment Patterns and Clinical Implications. Journal of Adolescence 21(4), 383-395.

Lewinsohn, P. M., Rohde, P. \& Seeley, J.R. (1996). Adolescent suicidal ideation and attempts: prevalence, risk factors, and clinical implications. ClinPsycholSciPract. ;325- 346.

National Mental Health Association \&The Jed Foundation. (2002). Safeguarding your students against suicide: Expanding the safety network. Alexandria, VA

Nerdrum, P., Rustøen, T., \&Rønnestad, M.H. (2006). Student psychological distress: A psychometric study of 1750 Norwegian, 1st-year undergraduate students. Scand. J. Educ. Res., 50(1), 95-109. 
Nolen-Hoeksema, S., Girgus, J. S., \& Seligman, M. E. (1991). Sex differences in depression and explanatory style in children. Journal of Youth and Adolescence, 20, 233-245.

Pelling, N., Bowers, R., \& Armstrong, P. (2007). The practice of counselling. Sydney: Thomson.

Roberts, R. E., Chen, Y.R. \& Roberts, C. R. (1997). Ethnocultural differences in prevalence of adolescent suicidal behaviors. Suicide Life Threat Behav. 27, 208- 217.

Stewart, S.M., Lam, T.H., Betson, C., \&Chung, S.F. (1999). Suicide ideation and its relationship to depressed mood in a community sample of adolescents in Hong Kong. Suicide and Life-Threatening Behavior,29(3),227-240.

Surgeon General of United States (2009). The Surgeon General's Call to Action to Prevent Suicide. US Public Health Service, Washington DC.

Talaei, A., Bordbar, F., Amir, R., \&Ardani (2009). Depression and its correlation with selfesteem and social support among Iranian university students. Iranian Journal of Psychiatry, 4(1), 17-22.

Velez, C. N. \& Cohen, P. (1988). Suicidal Behavior and Ideation in a Community Sample of Children: Maternal and Youth Reports. Journal of the American Academy of Child and Adolescent Psychiatry, 27 (3), 349-356.

World Health Organization (2010). Preventing Suicide. A resource for prison officers. Mental and Behavioural Disorders. Department of Mental Health.

Wright, L. S. (1985). Suicidal thoughts and their relationship to family stress and personal problems among high school seniors and college undergraduates. Adolescence, 20 (79), 575-580.

Yen, S., Shea, M.T., Pagno, M., Sanislow, C.A., \&Grilo, C.M. (2003). Axis I and axis II disorders as predictors of prospective suicide attempts: findings from the collaborative longitudinal personality disorders study. Journal of Abnormal Psychology, 112, 375381. 